\title{
First-principles study of electron transport through the single-molecule magnet $\mathrm{Mn}_{12}$
}

\author{
Salvador Barraza-Lopez ${ }^{1}$, Kyungwha Park ${ }^{1}$, Víctor García-Suárez ${ }^{2}$, and Jaime Ferrer ${ }^{3}$ \\ ${ }^{1}$ Department of Physics, Virginia Polytechnic \\ Institute and State University. Blacksburg VA, 24061 \\ ${ }^{2}$ Department of Physics, Lancaster University, \\ Lancaster, LA1 4YB, United Kingdom \\ ${ }^{3}$ Departamento de Fisica, Universidad de Oviedo, 33007 Oviedo, Spain
}

(Dated: November 24, 2018)

\begin{abstract}
We examine electron transport through a single-molecule magnet $\mathrm{Mn}_{12}$ bridged between $\mathrm{Au}$ electrodes using the first-principles method. We find crucial features which were inaccessible in model Hamiltonian studies: spin filtering and a strong dependence of charge distribution on local environments. The spin filtering remains robust with different molecular geometries and interfaces, and strong electron correlations, while the charge distribution over the $\mathrm{Mn}_{12}$ strongly depends on them. We point out a qualitative difference between locally charged and free-electron charged $\mathrm{Mn}_{12}$.

PACS numbers: 85.65.+h, 75.50.Xx, 85.75.-d, 73.23.Hk
\end{abstract}

\footnotetext{
* Present address: School of Physics, Georgia Institute of Technology, Atlanta, GA 30332
} 
In the past two decades, electron transport through quantum dots has been studied in single-electron transistors as an effort to manipulate single electrons at a time. [1, 2, 3] Semiconducting quantum dots were typically used because of easy manipulation of the number of electrons inside the dots, by varying gate voltages.

Recently, several experiments [4, 5, 6, 7] on electron transport through a single-molecule magnet $(\mathrm{SMM}) \mathrm{Mn}_{12}$ were reported in transistor set-ups or scanning tunneling microscope (STM) measurements. SMMs differ from magnetic clusters or quantum dots in the sense that transition metal ions in SMMs are interacting with each other via super-exchange through ligands, and that there is large magnetic anisotropy caused by spin-orbit coupling within each SMM. Thus, the degeneracy in different magnetic states for a given spin multiplet of SMMs is lifted even in the absence of external magnetic field. The main questions in these transport studies are whether the electronic and magnetic properties of SMMs would survive in low-dimensional structures and how the magnetic degrees of freedom interplay with the electronic degrees of freedom. The challenges in these types of experiments are, so far, to maintain stable molecular structures [8], to determine orientations of SMMs relative to surfaces, and to characterize interfaces.

Our previous first-principles studies [9, 10, 11] showed that SMMs are weakly coupled to $\mathrm{Au}$ surfaces and electrodes. Thus, transport through SMMs belongs to a Coulomb blockade regime. In most theoretical studies on transport through SMMs, one treated SMMs as quantum dots and relied on many-body model Hamiltonians with unknown parameter values. 12, 13, 14, 15, 16, 17] However, in contrast to quantum dots, the magnetic properties of SMMs are delicately balanced by interactions among transition metal ions. Thus, caution needs to be exercised in interpretation of experimental data to construct effective model Hamiltonians.

In this paper, we simulate semi-infinite electrodes and different molecular geometries and interfaces, and investigate transport properties through a $\mathrm{SMM} \mathrm{Mn}_{12}$ bridged between $\mathrm{Au}$ electrodes, using the non-equilibrium Green's function method in conjunction with spinpolarized density-functional theory (DFT). Our calculations provide crucial microscopic information such as a spin-filtering effect and a strong dependence of charge distribution over the $\mathrm{Mn}_{12}$ on local environments. This information was unattainable in the theoretical studies solely based on model Hamiltonians with unknown parameter values, and could qualitatively change transport properties through SMMs. $\mathrm{Mn}_{12}$ molecules used in the transport 

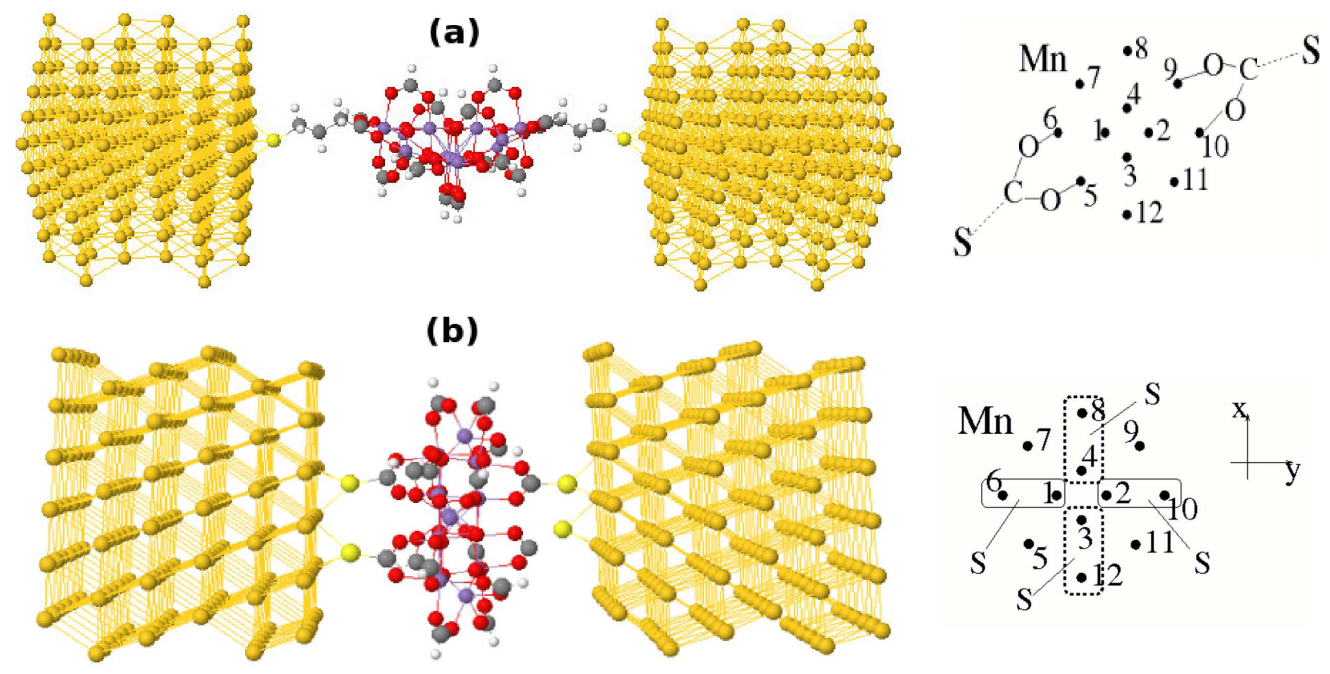

FIG. 1: (Color online) Scattering region for (a) geometry 1 consisting of $\mathrm{Mn}_{12}$ attached to $\mathrm{Au}$ layers via $\mathrm{S}$ atoms and alkane chains (distance between the electrodes $d=25.7 \AA$ ), and for (b) geometry 2 consisting of $\mathrm{Mn}_{12}$, four $\mathrm{S}$ atoms, and Au layers $(d=14.5 \AA)$. The transport direction is along the horizontal axis, $z$ axis. Semi-infinite Au electrodes are considered in calculations (not shown). On the right hand side the positions of the Mn ions are marked for each geometry. For geometry $\mathbf{1}$ the dashed lines indicate the alkane chains. For geometry $\mathbf{2}$ the solid and dashed blocks represent the areas where the four $\mathrm{S}$ atoms are attached through bonding to the $\mathrm{C}$ atoms.

experiments [4, 7] were bulky due to large ligands, and so the distances between the $\mathrm{Mn}_{12}$ molecule and the electrodes (or the lengths of linker molecules) for our molecular geometries are comparable to or much shorter than those in the experiments. We emphasize the effects of interfaces and molecular geometries on the charge distribution and the coupling constant between a SMM and electrodes. To take into account strong electron correlations in transition metal ions, we included a Hubbard-like $U$ term in our previous calculations. [10, 11]

We consider two molecular geometries for a $\mathrm{SMM} \mathrm{Mn}_{12}$ bridged between $\mathrm{Au}(111)$ electrodes as shown in Figs. 1 (a) and (b): (i) geometry 1 where the magnetic easy axis of $\mathrm{Mn}_{12}$ is perpendicular to the transport direction and $\mathrm{Mn}_{12}$ is attached to the electrodes via alkane chains and S atoms. (ii) geometry 2 where the easy axis of $\mathrm{Mn}_{12}$ is parallel to the transport direction and $\mathrm{Mn}_{12}$ is attached to the electrodes via four $\mathrm{S}$ atoms. The linker molecules are used to chemically bind the $\mathrm{Mn}_{12}$ to the electrodes, and play a role of energy barriers whose heights depend on their lengths and types of chemical bonding. The Au electrodes are 

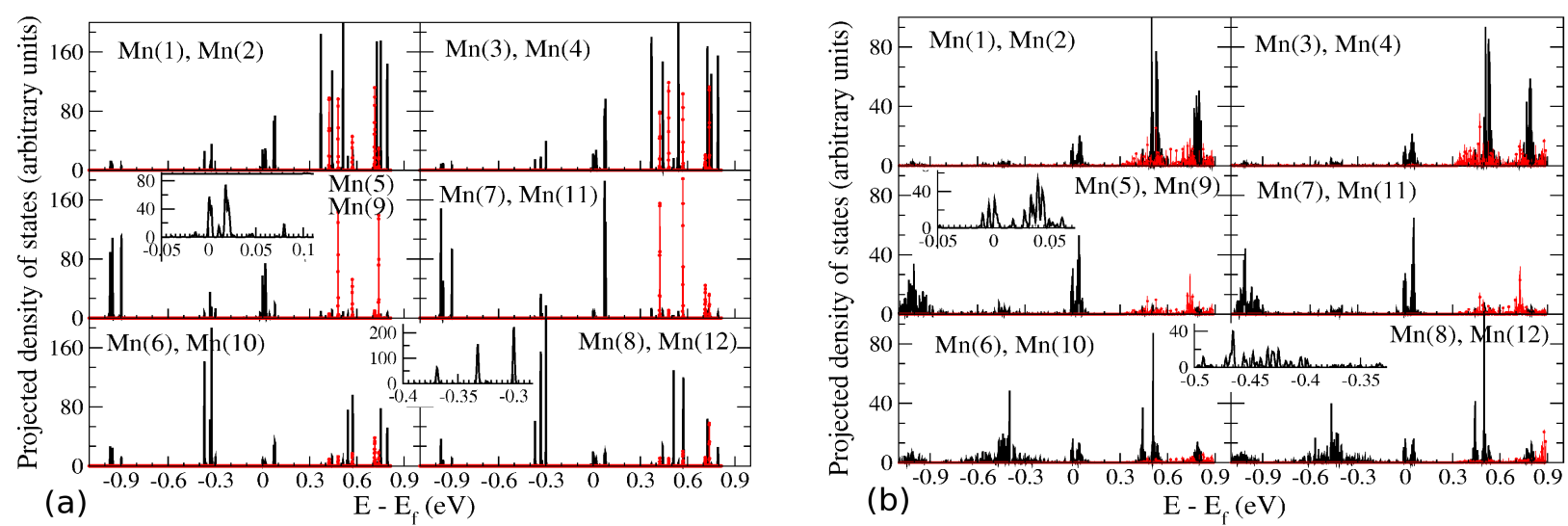

FIG. 2: (Color online) Spin-polarized density of states (DOS) projected onto Mn $d$ orbitals (a) for geometry 1 and (b) for geometry 2: majority spin (black), minority spin (red with symbol). Refer to Fig. 1 for numbering of the Mn ions. Insets: zoom-in of the majority DOS onto $\operatorname{Mn}(5), \operatorname{Mn}(9)$, $\operatorname{Mn}(8)$, and $\operatorname{Mn}(12)$.

treated as semi-infinite and the scattering region contains a few Au layers, linker molecules, and $\mathrm{Mn}_{12}$, as shown in Fig. 1. Our calculations are performed using SMEAGOL [18, 19], a quantum transport code interfaced with DFT SIESTA program [20]. Generalized-gradient approximation (GGA) [21] is used for exchange-correlation potential in spin-polarized DFT formalism. When spin-orbit coupling is included self-consistently for an isolated neutral $\mathrm{Mn}_{12}$ molecule, magnetic anisotropy barrier is computed to be $65.3 \mathrm{~K}$ using VASP[22] and 66.4 K using SIESTA, [19] which agrees well with experimental data.[23] Gate voltages and interactions with phonons are not considered in this study. Further details of the method and assumptions for this work and brief discussion on the transport for geometry $\mathbf{1}$ were presented in Ref. [11].

The spin-polarized densities of states (DOS) of the scattering region projected onto all Mn $d$ orbitals for geometries 1 and $\mathbf{2}$ are shown relative to the Fermi level, $E_{f}$, in Figs. 2(a) and (b). In the DOS for both geometries a bin size of $0.5 \mathrm{meV}$ and Gaussian broadening of $1 \mathrm{meV}$ are used. The minority-spin DOS become negligible in the energy window (-1.6, $0.42 \mathrm{eV})$ and $(-1.5,0.3 \mathrm{eV})$ relative to $E_{f}$ for geometries $\mathbf{1}$ and $\mathbf{2}$, respectively. Thus, for both geometries only the majority-spin orbitals contribute to the densities near $E_{f}$. Further discussion of the DOS for geometry $\mathbf{1}$ is followed by that for geometry $\mathbf{2}$.

For geometry 1 the fourfold symmetry of $\mathrm{Mn}_{12}$ is broken due to the linker molecules so that the degeneracy in the molecular orbitals is lifted. As shown in Fig. 2(a), the projected 
densities of states (PDOS) for $\mathrm{Mn}(5)$ and $\mathrm{Mn}(9)$ completely differ from those for $\mathrm{Mn}(7)$ and $\mathrm{Mn}(11)$, although the four Mn sites are equivalent according to the fourfold symmetry. The individual molecular orbitals are clearly identifiable due to the larger distance between the $\mathrm{Mn}_{12}$ and the electrodes (or longer linker molecules) compared to those in geometry 2. The coupling (or broadening) of the orbitals to the electrodes near $E_{f}$ is of the order of $1 \mathrm{meV}$ or less. The lowest unoccupied molecular orbital (LUMO) is located slightly above $E_{f}$ and the highest occupied molecular orbital (HOMO) is placed $0.29 \mathrm{eV}$ below $E_{f}$ [Fig. 2(a)]. The LUMO is mainly from two of the $\mathrm{Mn}$ ions in the outer ring, $\operatorname{Mn}(5)$ and $\operatorname{Mn}(9)$, while the HOMO is from $\mathrm{Mn}(8)$ and $\mathrm{Mn}(12)$.

For geometry 2 the shorter distance between the $\mathrm{Mn}_{12}$ and the electrodes allows the molecular orbitals to substantially broaden, which makes the individual orbitals unidentifiable [Compare the insets of Figs. 2 (a) and (b)]. That distance for geometry $\mathbf{2}$ is about a half of that for geometry $\mathbf{1}$, but the increase in the coupling constant for geometry $\mathbf{2}$ is much greater than a factor of 2 due to the exponential decay of the coupling with the distance for a given chemical bonding. The group of peaks near $E_{f}$ is formed by broadening of the LUMO, which arises from coupling of all of the Mn ions to the electrodes, in contrast to the case of geometry 1. The HOMO broadens due to coupling of $\mathrm{Mn}(6), \mathrm{Mn}(8), \mathrm{Mn}(10)$, and $\mathrm{Mn}(12)$ to the electrodes. In geometry 2 the fourfold symmetry of an isolated $\mathrm{Mn}_{12}$ is, to some extent, preserved, because the linker molecules are attached in a fourfold symmetric fashion. The coupling constant between the $\mathrm{Mn}_{12}$ and electrodes near $E_{f}$ is of the order of $10 \mathrm{meV}$ [insets of Fig. 2(b)].

We compute a spin-polarized transmission coefficient $T(E)$ for geometry $\mathbf{1}$ and our result at zero bias is shown in Fig. 3(a). The majority-spin LUMO is responsible for the resonant tunneling near $E_{f}$. The widths of the $T(E)$ peaks, in general, depend on broadening of the orbitals, phonon populations, and defects. In our case, since we did not include defects or interactions with phonons, the widths depend on the broadening only. The weak coupling leads to the widths of the $T(E)$ peaks ranging from 0.01 to $1 \mathrm{meV}$ [Fig. 3(b)]. The minorityspin contribution to $T(E)$ appears only $0.42 \mathrm{eV}$ above $E_{f}$ and $1.6 \mathrm{eV}$ below $E_{f}$. This agrees with the locations of the orbitals in the PDOS [Fig. 2(a)]. For geometry 1 there is a oneto-one mapping between the $T(E)$ peaks and the orbitals. The spatially resolved density of states integrated over $(-0.23,0.06 \mathrm{eV})$ relative to $E_{f}$ (Fig. 4) clearly corroborates that minority-spin electrons cannot tunnel through the $\mathrm{Mn}_{12}$ at low bias voltages. Using the same 

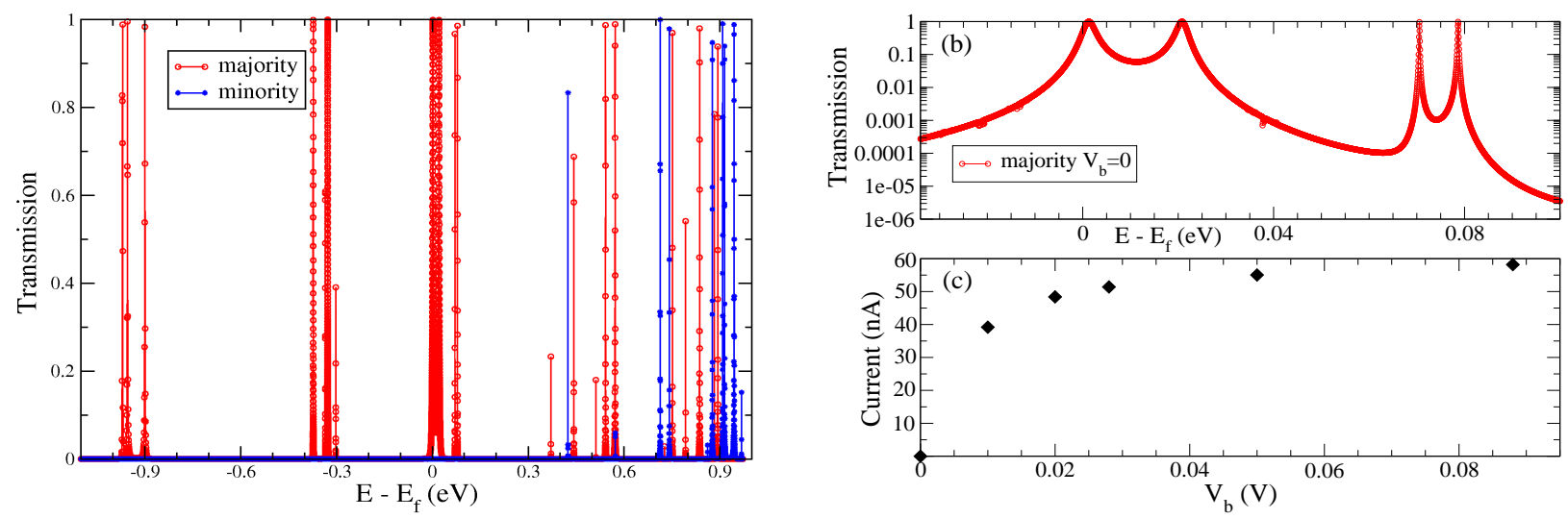

FIG. 3: (Color online) (a) Spin-polarized transmission coefficient at zero bias, (b) zoom-in of the majority-spin transmission at zero bias [(a)], and (c) computed current $I$ vs bias voltage $V_{b}$ for geometry 1.

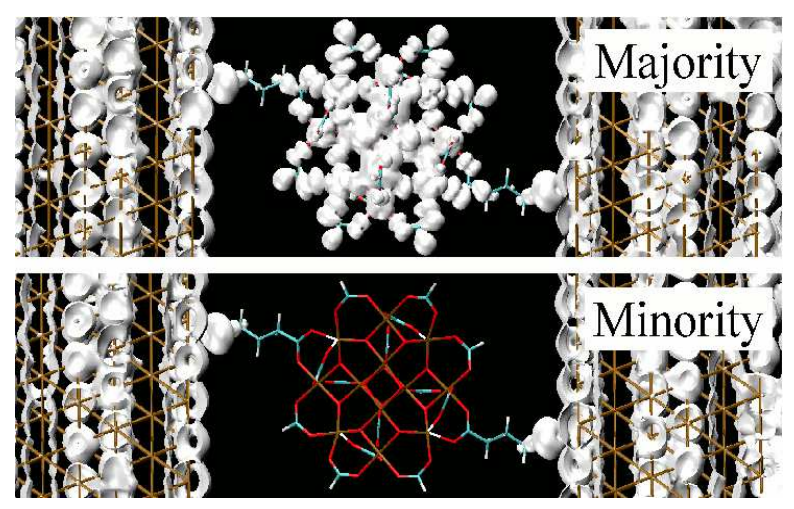

FIG. 4: (Color online) Majority-spin and minority-spin density of states integrated between -0.23 and $0.06 \mathrm{eV}$ relative to the Fermi level with isosurface criterion of $2 e / \mathrm{nm}^{3}$ for geometry 1 .

analogy, we expect that for geometry $\mathbf{2}$ only the majority-spin orbitals would contribute to $T(E)$ near $E_{f}$, and that the $T(E)$ peaks would be wider due to the broadening of the orbitals. As shown in Fig. 3(c), for geometry 1 our computed current as a function of bias voltage $V_{b}$ is of the order of tens of $\mathrm{nA}$ when $V_{b}<0.1 \mathrm{~V}$. For a given positive $V_{b}$, the chemical potential of the left (right) electrode increases (decreases) by $e V_{b} / 2$. We find that for $0<V_{b}<0.1 \mathrm{~V}$, $T(E)$ is shited upward compared to the zero-bias $T(E)$ without changing the main features. This shift is caused by the polarization of the $\mathrm{Mn}_{12}$ with $V_{b}$ and the amount of the shift is proportional to $V_{b}$. Then the area of $T(E)$ integrated over $E$ starts to saturate above $0.02 \mathrm{~V}$, which results in the saturation in the current-voltage dependence [Fig. 3(c)].

To compare with experiment, additional electron correlations within the Mn ions that are 


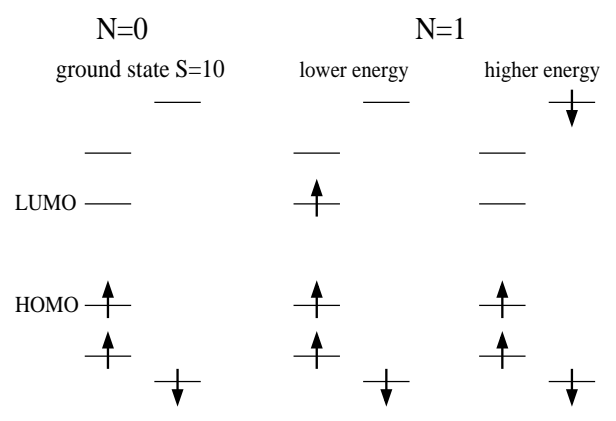

FIG. 5: Spin non-degenerate molecular orbitals of neutral $\operatorname{Mn}_{12}(N=0)$ in the ground state $(S=10)$ and of singly-charged $\mathrm{Mn}_{12}(N=1)$ in the ground state and an excited state, obtained from DFT. In each state, four majority-spin (two minority-spin) orbitals are shown on the left (right).

absent in standard DFT must be considered. Beyond DFT, the $U$ term was included in our calculation of the electronic structure of an isolated $\mathrm{Mn}_{12}$ molecule [10] using VASP. It was found that the majority-spin HOMO-LUMO gap increased to $1.1 \mathrm{eV}$ and that the majorityspin LUMO was shifted upward by $0.12 \mathrm{eV}$. The minority-spin LUMO was, however, still $0.12 \mathrm{eV}$ above the majority-spin LUMO. Thus, when an extra electron is added to the $\mathrm{Mn}_{12}$, the majority-spin orbitals are still well separated from the minority-spin orbitals. Consequently, only majority-spin electrons can be tunneled through the $\mathrm{Mn}_{12}$ at low bias voltages (below $0.5 \mathrm{eV}$ ). Thus, we conclude that the spin-filtering effect remains robust with different molecular geometries and interfaces, and strong electron correlations.

Henceforth, we discuss a subtle but important issue in the transport through $\mathrm{SMM} \mathrm{Mn}_{12}$. The computed charging energy of an isolated $\mathrm{Mn}_{12}$ is $3.8 \mathrm{eV}$ and thus the $\mathrm{Mn}_{12}$ can be only singly charged at low bias voltages. Due to the nature of less-than-half-filled $d$ orbitals, the Mn $d$ orbitals in the $\mathrm{Mn}_{12}$ are not spin-degenerate. If an extra electron is added to $\mathrm{Mn}_{12}$, the ground state would be achieved when the added electron has majority spin (Fig. 5). This is the origin of the spin-filtering effect in the transport through $\mathrm{Mn}_{12}$. However, this argument must not be straightforwardly interpreted that the ground-state spin of $\left[\mathrm{Mn}_{12}\right]^{1-}$, is $S=10+1 / 2=21 / 2$. This is true only if the ground-state spin of $\left[\mathrm{Mn}_{12}\right]^{1-}$ has a collinear configuration or if the extra charge is distributed over several Mn sites instead of being localized at one Mn site.

When conduction electrons are added to the $\mathrm{Mn}_{12}$ from the electrodes, our DFT calcu- 
lations with collinear spin configurations support that the electrons will be distributed over more than one Mn site. The specific distribution of the electrons over the Mn sites depends on the way the $\mathrm{Mn}_{12}$ molecule is attached to the electrodes. For geometry $\mathbf{1}$ the electrons will be mainly distributed over the $\operatorname{Mn}(5)$ and $\operatorname{Mn}(9)$ sites, while for geometry 2 they will be distributed over all of the Mn sites [Figs. 2(a) and (b)]. Our noncollinear DFT calculations on $\left[\mathrm{Mn}_{12}\right]^{1-}$ (using SIESTA) reveal that the collinear spin configuration with $S=21 / 2$ has the lowest energy.

Some transport studies based on model Hamiltonians, [4, 13, 16] assumed that a singly charged $\mathrm{Mn}_{12}$ molecule has the ground-state spin of $S=19 / 2$, by referring to experiments [24, 25] performed on locally charged $\mathrm{Mn}_{12}$ molecules. In these experiments, extra electrons were added to the magnetic core of the $\mathrm{Mn}_{12}$, by inserting cations (or electron donors) close to one or two of the Mn ions. Since a bulk form of $\mathrm{Mn}_{12}$ molecules is an insulator, the added electrons would be localized to the Mn sites closest to the cations. The experiments showed that the total spin for $\left[\mathrm{Mn}_{12}\right]^{-1}$ was $S=19 / 2$, and that one of the $\mathrm{Mn}$ ions in the outer ring changed its valence from $3+(S=2)$ to $2+(S=5 / 2)$. In contrast to typical quantum dots, the total spin of $\mathrm{Mn}_{12}$ is maintained through delicate balance among interactions between the different $\mathrm{Mn}$ ions. We perform noncollinear calculations on $\mathrm{Mn}_{11} \mathrm{Fe}$ (one of the $\mathrm{Mn}$ ions in the outer ring is replaced by $\mathrm{Fe}$ in the $\mathrm{Mn}_{12}$ geometry) in order to mimic a singly locally charged $\mathrm{Mn}_{12}$. Using $U_{\mathrm{Mn}}=4 \mathrm{eV}$ [10] and $U_{\mathrm{Fe}}=6 \mathrm{eV}$ in VASP, we find that the collinear spin configuration with $2 S=19$ has $100 \mathrm{meV}$ higher energy than the collinear spin configuration with $2 S=21$. The latter has $5.8 \mathrm{meV}$ higher energy than the noncollinear spin configuration $(2 S=18.71)$ in which the magnetic moment vector of the $\mathrm{Fe}^{3+}(S=5 / 2)$ ion is tilted by $62^{\circ}$ from the moment vectors of the eleven Mn ions mostly aligned along with the $z$ axis. Additional noncollinear calculations on the $\mathrm{Mn}_{11} \mathrm{Fe}$ with $U=0$ also supports that the collinear spin configuration with $2 S=21$ is not the ground state for the singly locally charged $\mathrm{Mn}_{12}$. Thus, the spin-filtering effect and single-electron picture (Fig. 5) are compatible with the experimental findings [24, 25].

In summary, we have investigated transport properties through a $\mathrm{Mn}_{12}$ molecule bridged between $\mathrm{Au}(111)$ electrodes using the non-equilibrium Green's function method and spinpolarized DFT. We found that the $\mathrm{Mn}_{12}$ functioned as a spin filter in a low bias regime (below $0.5 \mathrm{eV})$. The spin-filter effect persisted with different molecular geometries and interfaces and strong electron correlations, while the distribution of conduction electrons over the $\mathrm{Mn}_{12}$ 
strongly depended on them. Conduction electrons from the electrodes would be distributed over several Mn sites rather than being localized at one Mn site. There is no contradiction between the spin-filtering effect and the experimental observation on $S=19 / 2$ for the singly locally charged $\mathrm{Mn}_{12}$ molecules.

K.P. was supported by NSF DMR-0804665, the Jeffress Memorial Trust Funds, and NCSA under DMR060009N. J.F. was supported by MEC FIS2006-12117. The authors are grateful to W. Wernsdorfer for discussions.

[1] Y. Meir et al., Phys. Rev. Lett. 66, 3048 (1991).

[2] D. Goldhaber-Gordon et al., Nature 391, 156 (1998).

[3] S. M. Cronenwett et al., Science 281, 540 (1998).

[4] H. B. Heersche et al., Phys. Rev. Lett. 96, 206801 (2006)

[5] M.-H. Jo et al., Nano Lett. 6, 2014 (2006).

[6] J.J. Henderson et al., J. Appl. Phys. 101, 09E102 (2007).

[7] S. Voss et al., Phys. Rev. B 78, 155403 (2008).

[8] A recent report [M. Mannini et al., Nature Materials 8 194, (2009)] on a thin film of SMMs $\mathrm{Fe}_{4}$ showed a possibility of stabilization of SMMs on surfaces.

[9] S. Barraza-Lopez et al., Phys. Rev. B 76, 224413 (2007).

[10] S. Barraza-Lopez et al., J. Appl. Phys. 103, $07 B 907$ (2008).

[11] S. Barraza-Lopez et al., J. Appl. Phys. 105, $07 E 309$ (2009).

[12] G.-H. Kim and T.-S. Kim, Phys. Rev. Lett. 92, 137203 (2004).

[13] C. Romeike et al., Phys. Rev. Lett. 96, 196805 (2006).

[14] M. N. Leuenberger and E. R. Mucciolo, Phys. Rev. Lett. 97, 126601 (2006).

[15] F. Elste and C. Timm, Phys. Rev. B 73, 235305 (2006).

[16] G. González et al., Phys. Rev. B 78, 054445 (2008).

[17] L. Michalak et al., arXiv:0812.1058

[18] A.R. Rocha et al., Phys. Rev. B 73, 085414 (2006).

[19] L. Fernández-Seivane et al., J. Phys.: Condens. Matter 18, 7999 (2006).

[20] J. M. Soler et al., J. Phys.: Condens. Matter 14, 2745 (2002); J. Junquera et al., Phys. Rev. B 64, 235111 (2001); P. Ordejón et al., Phys. Rev. B 51, 1456 (1995). 
[21] J. P. Perdew et al., Phys. Rev. Lett. 77, 3865 (1996).

[22] G. Kresse and J. Furthmüller, Phys. Rev. B 54, 11169 (1996); G. Kresse and J. Furthmüller, Comp. Mat. Sci. 6, 15 (1996).

[23] A. L. Barra et al., Phys. Rev. B 56, 8192 (1997); S. Hill et al., Phys. Rev. Lett. 80, 2453 (1998).

[24] H. J. Eppley et al., J. Am. Chem. Soc. 117, 301-317 (1995); M. Soler et al., J. Am. Chem. Soc. 125, 3576 (2003).

[25] R. Basler et al., Inorg. Chem. 44, 649 (2005). 